\title{
陆地生物圈模型的发展与应用
}

\author{
彭书时 ${ }^{1^{*}}$ 岳 超 常锦峰 $^{3}$ \\ ${ }^{1}$ 北京大学城市与环境学院, 北京大学地表过程分析与模拟教育部重点实验室, 北京 $100871 ;{ }^{2}$ 西北农林科技大学水土保持研究所, 陕西杨凌, 712100 ; \\ ${ }^{3}$ International Institute for Applied Systems Analysis (IIASA), A-2361 Laxenburg, Austria
}

摘 要 陆地生物圈与大气圈和水圈之间能量、水和碳氮等元素的交换和循环对整个地球系统产生了深刻的影响。陆地生物 圈模型(TBM) 是研究陆地生态系统如何响应和反馈全球变化的重要方法和工具。通过对从生态系统到区域和全球陆地生物圈 不同空间尺度的植被动态、生物地球物理和生物地球化学循环过程、水循环和水文过程、自然干扰和人类活动等过程时间动 态的模拟, 陆地生物圈模型被广泛地应用于评估和归因过去陆地生物圈的时空变化和预测陆地生物圈对未来全球变化的响 应和反馈。该文简要回顾了陆地生物圈模型的发展, 总结了模型对陆地生态系统主要过程的刻画和模型在生态系统生态学的 应用, 并对未来陆地生物圈模型的发展和应用进行了展望。

关键词 陆地生物圈模型; 陆地生物圈; 生态系统生态学; 生态系统研究; 全球变化; 碳循环

彭书时, 岳超, 常锦峰 (2020). 陆地生物圈模型的发展与应用. 植物生态学报, 44, 436-448. DOI: 10.17521/cjpe.2019.0315

\section{Developments and applications of terrestrial biosphere model}

\author{
PENG Shu-Shi ${ }^{*}$, YUE Chao ${ }^{2}$, and CHANG Jin-Feng ${ }^{3}$
}

${ }^{1}$ College of Urban and Environmental Sciences, Key Laboratory for Earth Surface Processes of Ministry of Education, Peking University, Beijing 100871, China; ${ }^{2}$ Institute of Soil \& Water Conservation, Northwest A\&F University, Yangling, Shannxi 712100, China; and ${ }^{3}$ International Institute for Applied Systems Analysis (IIASA), A-2361 Laxenburg, Austria

\begin{abstract}
Exchanges of energy and matter between terrestrial biosphere and atmosphere and hydrosphere create critical feedbacks to Earth's climates. To quantify how terrestrial ecosystems respond and feedback to global changes, terrestrial biosphere model (TBM) has been developed and applied in global change ecology during the past decades. In TBMs, myriad of biogeophysical, biogeochemical, hydrological cycles and dynamics processes on different spatial and temporal scales are represented. The TBMs have been applied on assessing and attributing past changes in terrestrial biosphere, and on predicting future changes and their feedbacks to climates. Here, we provide an overview of processes included in TBMs and TBMs applications on carbon and hydrological cycles, as well as their application on exploring human impacts on terrestrial ecosystems. Finally, we outline perspectives for future development and application of TBMs.
\end{abstract}

Key words terrestrial biosphere model; terrestrial biosphere; ecosystem ecology; ecosystem research; global change; carbon cycle

Peng SS, Yue C, Chang JF (2020). Developments and applications of terrestrial biosphere model. Chinese Journal of Plant Ecology, 44, 436-448. DOI: 10.17521/cjpe.2019.0315

陆地生物圈是地球系统的重要圈层, 其与大气 圈层和水圈之间能量、水、碳氮等化学要素的交换 和相互作用深刻影响了地球气候, 同时陆地生物圈 对气候变化也很敏感(Prentice et al., 2007)。随着全 球变化科学的不断发展, 对陆地生物圈如何响应和 反馈气候变化的认知也不断深入, 预测未来陆地生 物圈的变化和其对地球气候的反馈逐渐成了全球变
化科学核心科学问题。2005年《Science》杂志评选 出125个关键未解的科学问题也包括了陆地生态系 统对全球变化如何响应和未来地球温度会升高多少 这两个问题。陆地生物圈模型(TBM)在评估过去陆 地生物圈的变化及其归因和预测未来陆地生物圈对 气候变化的响应中发挥了重要作用(IPCC, 2013), 也成为了研究区域和全球尺度陆地生态系统和环境 
变化问题的重要方法。

已有研究表明未来气候变化预测的不确定性有 一半来自于陆地生物圈, 例如陆地生物圈碳循环对 气候变化的响应与反馈(Friedlingstein et al., 2006, 2014; Bodman et al., 2013)。由于地球气候对陆地生 态系统的变化非常敏感, 从IPCC第一次评估报告到 第五次评估报告, 陆地生物圈模型也逐步成为地球 系统模式中的关键部分(IPCC, 2013)。发展陆地生物 圈模型和降低陆地生物圈模型的不确定性也被认为 是准确预测未来地球气候变化的关键(Fisher et al., 2014)。陆地生物圈模型不仅是生态系统生态学研究 的重要工具, 也是地球系统模式中的重要模块。近 20年陆地生物圈模型在国际地圈与生物圈计划 (IGBP)、全球环境变化计划(GECP)等多个国际项目 的推动下, 融合了生态学、环境科学和地球系统科 学等多学科基础和交叉前沿, 迅速发展并形成了耦 合多种过程的复杂模型。为了推动陆地生物圈模型 的进一步发展和与生态学有关的应用, 本文将简要 回顾陆地生物圈模型的发展历史, 对陆地生物圈模 型的主要过程进行简介, 总结模型在生态系统生态 学中的应用, 并展望模型未来的研究方向。

\section{1 陆地生物圈模型发展历史}

自1849年亚历山大·冯·洪堡 (Alexander von Humboldt)首次建立气候与植物地理关系后, 经奧 古斯特·格里瑟巴赫(August Grisebach)和弗拉迪米 尔·彼得·柯本(Wladimir Peter Köppen)细化完善后, 形成了植物地理预测模型的维形。植物地理模型能 够描述气候平衡态植被类型的静态空间分布, 但不 能模拟碳氮水循环, 也不能模拟能量等生物物理过 程。与大尺度植物地理模型相反, 描述小尺度植被 动态的模型主要关注植物个体生长和死亡, 对光、 水和营养元素的竞争和群落演替等过程, 这些小尺 度模型逐渐模拟了小尺度上森林植被动态(Watt, 1947), 例如HYBRID和ED模型(Friend et al., 1997; Moorcroft et al., 2001)。这些小尺度植被动态模型一 般不能保证物质或能量守恒, 随后发展的生物地球 化学循环模型建立在物质和能量守恒的基础上, 研 究碳氮水在生态系统中的循环过程, 例如 CENTURY模型(Parton et al., 1994)。同时, 早期为了 模拟陆地与大气边界层之间能量、水汽和动量过程, 与气候和大气环流模型(GCMs)相耦合, 陆面模式
(LSMs)基于生物物理模型的基础应运而生, 例如 SiB模型(Sellers et al., 1997)。早期的陆面模式侧重 陆地生态系统生物物理过程的模拟, 缺乏植被动态 和生物地球化学循环过程。关于这些不同类型的模 型定义和区分可进一步参考Fisher等(2014)文中的 表1。

在 20 世纪 80 年代之前, 上述 4 种模型基本上是 独立发展。1988-1990年间, 由国际应用系统研究所 (IIASA) 所领导的生物圈动态研究项目举办了一系 列会议, 号召国际科学界整合4种模型发展全球动 态植被模型(DGVMs), 用于模拟生态系统的动态及 物质和能量的流动。随后国际地圈生物圈计划 (IGBP)全球变化与陆地生态系统工作组(GCTE)采 纳了 DGVMs 的方案, 并提供了发展框架。随着 DGVMs的不断发展和更多过程的加入(例如水文模 型), DGVMs 广义上也被称为陆地生物圈模型 (Fisher et al., 2014)。为了理解和预测自然干扰和人 为活动对陆地生物圈的影响, 陆地生物圈模型进一 步耦合了自然干扰模块(火灾、台风)和人类活动模 块(土地利用变化、农作物、森林和草地管理等模 块)。综上所述, 陆地生物圈模型的发展融合了生物 地球物理学、水文过程、生物地球化学循环、植被 动态、自然干扰和人类活动等主要过程(图1)。目前 参与国际多模型比较项目的陆地生物圈模型主要有 LPJ、CLM、ORCHIDEE、CABLE、DLEM、JULES 等, 详细模型列表可参考Huntzinger等(2013)和Sitch 等(2015)的文献。附录I列出了同时参与了国际陆地 生物圈模型比较项目MsTMIP和TRENDY的模型及 其参考文献。

\section{2 陆地生物圈模型过程简介}

近20年陆地生物圈模型的发展囊括了不同时空 尺度的过程, 包括叶片尺度的气孔、光合和蒸腾, 冠 层尺度的物候、冠层温度、降水截留和空气动力学 特征, 植物尺度的穿透雨、树干径流、自养呼吸、 净初级生产力和分配, 生态系统尺度的土壤温湿 度、碳库动态、氮磷吸收、分解和异氧呼吸、净生 态系统生产力、蒸散发、入渗、径流、植物功能型 竞争、生长、死亡和干扰等过程。陆地生物圈模型 通过参数化的数学方程描述这些不同尺度的过程在 小时、天、月和年等时间尺度上的动态。陆地生物 圈模型对这些过程的定量描述毫无疑问依赖于对生 


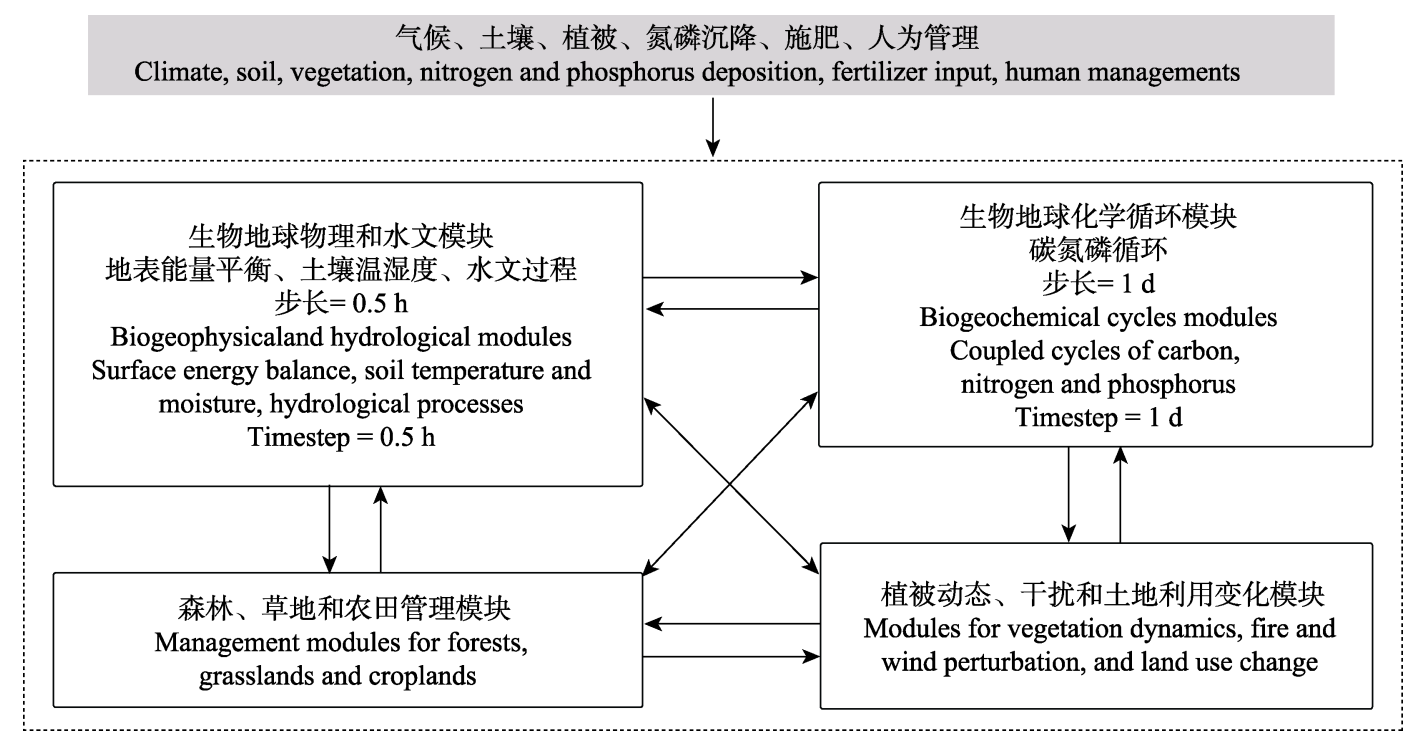

图1 陆地生物圈模型框架图(以ORCHIDEE模型为例)。

Fig. 1 Framework of terrestrial biosphere model taking ORCHIDEE as an example.

态系统生态学认知的不断深入。

虽然不同的陆地生物圈模型对不同过程的参数 化方案或算法有所不同，但基本框架和结构是类似 的(Huntzinger et al., 2013; Fisher et al., 2014)。本节 以法国的陆地生物圈模型ORCHIDEE为例, 简要介 绍模型中生物地球物理过程, 水文过程, 生物地球 化学循环, 植被动态、干扰和土地利用模块, 森林、 草地和农田管理模块(图1)。ORCHIDEE默认版本 (trunk)及不同开发版本(branch)的代码下载和使用 说明文档请见该模型网站http://forge.ipsl.jussieu.fr/ orchidee/wiki。

ORCHIDEE模型基于植物功能型(PFT)来模拟 以上过程。ORCHIDEE默认版本有 12 个PFT, 分别为 热带雨林、热带季雨林、温带常绿针叶林、温带常 绿阔叶林、温带落叶阔叶林、北方常绿针叶林、北 方落叶阔叶林、北方落叶针叶林、 $\mathrm{C}_{3}$ 草地、 $\mathrm{C}_{4}$ 草地、 $\mathrm{C}_{3}$ 农作物、 $\mathrm{C}_{4}$ 农作物; 此外还有一类为裸地类型。 用户也可以自己在模型中定义PFT。ORCHIDEE模 型的主要驱动数据有气候(向下短波和长波辐射、近 地面空气温湿度、降雨和降雪、风速和大气压), 土 壤和植被; 氮磷模块需要输入氮磷沉降和施肥数据, 森林、草地和农田管理模块需要输入相应的人为管 理数据(图1)。

\section{1 生物地球物理过程}

ORCHIDEE模型的生物地球物理过程主要包 括地表能量平衡和土壤热传导。地表能量平衡模块
根据输入的向下短波和长波辐射、风速、空气温湿 度以及地表状态计算出向上短波和长波辐射、感热 和潜热通量，以及土壤热通量(Ducoudré et al., 1993; de Rosnay \& Polcher, 1998)。为了计算土壤热传导和 土壤温度, 用户可以设置土壤热传导深度和分层厚 度; 根据热传导偏微分方程和其傅里叶求解方法, 土壤热传导深度一般设置较深, 默认土壤热传导深 度为 $90 \mathrm{~m}$, 按照指数增加的间距分为 46 层。其中每 层土壤的热传导率和热容量与土壤质地和土壤含水 量有关, 每30 min计算出每层土壤的热通量和土壤 温度。用户可以设置土壤热传导底部的地热通量和 是否考虑深层基岩热通量。

\section{2 水文过程}

ORCHIDEE模型的水文过程包括了降水分配、 蒸散、地表和土壤水分平衡三部分。降水包括了降 雪和降雨。对降雪向生态系统水分输入的模拟较为 简单，即不考虑草本和木本植被由于高度不同而导 致的各自积雪覆盖情况，而是假设所有降雪直接落 在地表。雪的融化或堆积取决于雪面的能量平衡状 况，降雪或积雪产生的融水进入地表水。降雨分配 包括冠层截流和到达地表的穿透雨, 其中冠层截流 占降雨量的比例取决于植物功能型。植被冠层最大 持水能力是叶面积指数的函数, 根据冠层当前持水 量和最大持水能力可以计算得出干燥和湿润冠层的 相对比例, 并假设蒸腾过程仅仅发生在干燥冠层部 分, 而湿润冠层部分水分散失先受蒸发过程主导 
(即冠层截流蒸发), 待冠层水分消耗殆尽后, 水分 散失变为蒸腾过程。

水分从生态系统向大气边界层的散失即是蒸散 发过程。ORCHIDEE对蒸散发过程的模拟整体上采 用了潜在蒸散发除以水分散失所遇到的阻抗的形 式。其中, 蒸散发过程包括了雪的升华、冠层截流 蒸发、冠层蒸腾以及地表蒸发。冠层截流蒸发仅发 生在湿润冠层部分, 其阻抗包括冠层结构阻抗和空 气动力学阻抗; 冠层蒸腾表征了水分通过敞开的叶 片气孔由植物体内迁移到大气边界层的过程, 其阻 抗包括叶片气孔阻抗、冠层结构阻抗和空气动力学 阻抗; 地表蒸发阻抗包括表层土壤阻抗和空气动力 学阻抗。

地表水分平衡包括了由穿透雨和积雪融水形成 的输入以及由入渗和地表径流形成的输出, 当水分 输入大于入渗时, 就会形成地表径流。在个别坡度 较低区域, 地表径流会再次向土壤入渗(再入渗过 程), 构成地表水输入的第三个来源。在每个格点内, 土壤水库按照植被类型分为相互独立的三个部分: 木本、草本和裸地, 即水分平衡在每部分独立核算。 默认土壤深度为 $2 \mathrm{~m}$, 按照指数增加的间距分为 11 层。地表蒸发仅仅发生在最上层土壤, 该层以下的 土层湿度变化取决于垂向水分交换和蒸腾量。土层 间垂向水分交换由描述一维非饱和土壤水分运动的 Fokker-Planck方程进行模拟(de Rosney et al., 2002), 不同格点间的水分横向运动予以忽略, 后者在大尺 度上应用模型时是适当的。根据不同土层的根系分 布状况和土壤湿度, 将植被蒸腾消耗的水量分配至 各土壤层(Seidl et al., 2017)。最下层土壤水分饱和时 会形成重力排水, 重力排水和地表径流一起, 构成 河川径流。

\section{3 生物地球化学循环}

\subsection{1 碳循环}

ORCHIDEE对碳循环的模拟主要包括生态系 统植被、调落物和土壤碳库、各碳库之间的碳通量 和生态系统与大气之间的碳通量。默认版本中碳库 包括8个生物量碳库(叶、根、地上茎、地下茎、地 上心材、地下心材、花和果实、储藏库)、6个调落 物碳库和 3 个土壤碳库。调落物碳库根据调落物的来 源分成了地上和地下各 3 个周转周期的碳库; 土壤 碳库的 3 个碳库分别是活性碳库、缓性碳库和惰性碳 库(Krinner et al., 2005)。光合作用模拟采用Ball
Berry的计算方案(Yin et al., 2009), 光合作用的产物 通过分配进入植物生物量碳库的各个部分, 同时植 物进行自养呼吸(包括生长呼吸和维持呼吸)向大气 中释放 $\mathrm{CO}_{2}$; 而植物的器官调落或植物死亡后, 进 入调落物碳库; 调落物一部分经过分解以 $\mathrm{CO}_{2}$ 的形 式释放到大气中, 剩下的部分进入土壤的 3 个碳库。 土壤3个碳库通过分解作用在 3 个碳库之间重新分配, 并向大气释放 $\mathrm{CO}_{2}$ 。凋落物和土壤碳库的分解为异 养呼吸。物候模块控制着植物光合作用开始和结束 的时间及叶片的寿命。

\subsection{2 氮磷循环}

植物、土壤及微生物的元素化学计量关系相对 稳定使得碳循环与营养元素循环(例如氮循环和磷 循环)紧密耦合在一起。这一耦合机制使得陆地生态 系统碳循环对全球变化的响应在一定程度上取决于 气候变化下营养元素的可利用性(Zaehle et al., 2015)。 因此, 为了更好地模拟陆地生态系统对全球变化的 响应, ORCHIDEE陆续加入了氮(ORCHIDEE-CN)和 磷 (ORCHIDEE-CNP) 循环 (Goll et al., 2017)。 ORCHIDEE-CN模型版本模拟了陆地生态系统的主 要氮循环过程及其与碳循环的耦合, 主要包含: 基 于氮丰度的叶片光合作用、自养呼吸及其对植被冠 层碳水通量影响的模拟; 植物氮吸收; 考虑植物不 稳定和稳定储藏库动态包括生长季末期氮重吸收, 以改进模型对物候及生长速率的模拟; 考虑植物氮 含量对异速生长比例(生物量分配)及根、枝干与叶 之间比例的限制; 土壤氮动态(包括氮矿化、硝化、 反硝化作用及氮相关气体的排放和淋溶等)和生物 固氮作用等。ORCHIDEE-CNP模型版本能够进一步 考虑植物体内可利用磷对植物生长的限制, 并模拟 植被磷需求、吸收、分配和周转(包括重吸收); 磷在 植物体和土壤中的生物化学矿化作用; 自然界磷风 化; 土壤磷动态(包括土壤磷移动性、土壤吸附、淋 溶等); 根际磷动态; 以及微生物和植物对有效磷的 竞争等过程。

\section{4 植被动态、干扰和土地利用变化}

陆地生态系统时时刻刻处于动态变化中, 这些 变化有些是渐进式的, 如自然因素变化(气温、降 水、大气 $\mathrm{CO}_{2}$ 浓度等)导致的植被类型组成的变化; 有些是迸发式的, 如影响较大的野火干扰、森林风 灾、虫灾等。此外, 人为活动导致的土地利用变化 也是影响植被动态的重要因素。ORCHIDEE模型将 
这些植被变化进行了区分, 目前能够模拟：(1)环境 因素变化导致的植物功能群组成的变化(简述为植 被动态); (2)野火干扰和风灾; (3)土地利用变化。

环境因素对植被动态的影响包括了植物的更 新、生长和死亡, 这些过程同时也决定了植物个体 对资源的竞争, 从而导致植被功能性的动态变化 (Sitch et al., 2003)。植物生长过程(光合作用、净初 级生产力的形成及分配) 是碳循环过程的一部分, 这里重点介绍更新和死亡过程。对植物更新过程的 模拟包括: 在合适的气候条件下, 对木本和草本植 物作为一个整体各自设定一个潜在更新速率。木本 植物潜在更新速率随投射盖度增加而下降, 以反映 现有木本植物盖度通过改变林下辐射状况而影响树 木幼苗的更新; 草本植物的潜在更新速率取决于潜 在最大植被盖度与现有植被盖度的差值, 即反映可 供幼苗更新利用的空间大小的影响。在此基础上, 木本或草本植被内部不同植物功能型的更新速率取 决于植物的迁移能力和长期生产力, 以反映不同功 能型的竞争能力。ORCHIDEE模型植被动态模块中 植物个体对资源的竞争模型来源于LPJ模型, 详细 过程和方程可参考Sitch等(2003)。

ORCHIDEE中由非干扰因素导致的死亡机制 包括: 由光竞争导致的死亡, 植物自身生长效率过 低从而无法维持其生理活动导致的死亡, 植物无法 适应气候。当木本植被盖度之和超过一个阈值 (0.95)或所有植被盖度之和超过1时, 所有植物功能 型都将由于辐射不足(光竞争)而发生部分死亡, 死 亡率与该功能型当年盖度增加幅度正相关。植物生 长效率定义为当年生物量增量除以叶面积, 死亡率 与生长效率成反比; 当某一功能型过去 3 年的平均 净初级生产力 $(N P P)$ 为负时, 该功能型所有个体将 全部死亡。此外, ORCHIDEE通过引入某些植物功 能型自然分布的气候界限, 决定该功能型能否更新 及存活, 例如限制植被存活更新和分布的最冷月平 均气温的多年极端最小值和最大值等。对于落叶阔 叶林, 其死亡率还受到春季霜冻过程的影响。

除环境气候因素外, 干扰是导致植被死亡的另 一重要影响因素。ORCHIDEE包括了火灾和风灾两 种干扰形式。火灾的发生受可燃物量、可燃物干燥 程度、气象条件(温度、湿度、风速等)和着火源的 影响。ORCHIDEE对火扩散速度的模拟基于具备实 验基础的准经验 Rothermel模型(Rothermel, 1972),
该模型考虑了火释放能量向周围燃料的传递以及可 燃物达到燃点所需能量间的对比关系。火灾导致树 木的死亡率包括了冠层直接灼烧和高温导致树木形 成层受损两种比例。在另外一个能够解析森林冠层 结构的ORCHIDEE版本中, 加入了风灾对森林的影 响。根据森林冠层结构特征(冠层高度、树干湿重) 和大气边界层的空气动力学特征(表面粗糙度、零平 面位移), 可以计算得出整株倒伏和树干折断所需 的临界风速。风速达到临界风速时, 就会造成树木 死亡, 死亡率与风速增加的关系成S形曲线。对于天 然林, 风倒植物残体将进入调落物库进行分解; 对 于人工管理的森林, 模型可以进一步模拟挽救性采 伐, 后者通常用以防止风灾后大量倒木所促成的虫 灾爆发。

人类通过土地利用变化和生态系统管理大幅改 变了地表植被组成, 因此这些活动也应纳入陆地生 物圈模型中。ORCHIDEE模型能够模拟土地利用变 化、森林管理以及农田和草场管理, 其中对土地利 用变化的模拟需要历史时期土地类型转换的驱动数 据, 可以包括土地覆盖类型的净变化(如毁林造田)、 同一模型格点内两种土地利用类型间的等量双向变 化(如热带地区森林-农田轮转的游耕农业) 以及森林 木材砍伐。热带地区的毁林造田中, 火常常被用来 清除地表自然干化后的森林生物量, 模型通过将该 生物量的一部分在毁林当年作为 $\mathrm{CO}_{2}$ 的形式释放到 大气中进行模拟, 剩余的生物残体进入调落物库进 行分解。以木材获取为目标的森林砍伐所获得的木 材产品被分配到存续时间不同(1、10和100年)的碳 库中, 以描述不同用途的木产品(纸张、家具、建筑 等)将其所含的碳归还到大气中所需的不同时间。

\section{5 森林、草地和农田管理模块}

\subsection{1 森林结构和森林管理}

在大尺度陆地生物圈模型中表征森林结构和森 林管理主要出于两方面的原因: 一是气候变化背景 下, 随着火灾、风灾、干旱、虫灾等导致森林局部 受损干扰事件的增多, 森林冠层结构变得复杂, 原 有的大叶模型无法准确刻画森林动态以及冠层结构 变化对地表过程的影响; 二是森林管理活动(间伐、 皆伐、实生林、萌生林、轮作周期影响等)对气候和 环境影响变得日益重要。ORCHIDEE-CAN模型版本 大幅完善了对森林结构及相关地表过程的模拟, 主 要包括了以下几个方面: 
(1)能够解析冠层结构。模型可以明确表征森林 密度及森林年龄结构, 后者通过将树木按照胸围分 成不同的径级组实现。净光合产物的分配遵循经典 的管道模型所描述的树木几何学特征, 个体较大的 树由于获取资源较多(光截获、养分获取等), 所分配 的净光合产物也多, 生长也更快。这些可以很好地 描述不同个体间的竞争过程。

(2)能够描述树木的水力结构。大叶模型中, 土 壤水分通过限制最大羧酸化光合速率影响光合过程, 蒸腾量在对冠层能量平衡和光合作用过程的模拟中 一并计算。而在考虑森林结构的ORCHIDEE-CAN 模型中, 对蒸腾过程模拟考虑了树木的水力结构, 即蒸腾量由土壤-叶片水势差和根-树干-叶片气孔水 分传输系统的水力阻抗共同决定。当通过水力结构 计算的允许蒸腾量小于能量平衡得出的蒸滕量时, 能量平衡过程将重新计算, 通过多次迭代最后获得 与水力结构相吻合的冠层能量平衡状态和对应的光 合量。

(3)冠层结构、辐射传输与多层能量平衡

在ORCHIDEE-CAN中, 树木冠层用球体表征, 树木相对空间位置用泊松分布表征, 这些特征可以 用来计算沿冠层剖面的林窗和辐射分布情况, 冠层 反照率不再是外部给定的静态量, 而是根据冠层结 构特征通过双向辐射传输模型动态计算获得(Ryder et al., 2016)。在此基础上, 可以获得叶面积密度沿 冠层剖面的分布, 并将冠层分解成多层结构, 为每 层单独进行能量平衡计算, 因此最终叶面积指数由 碳收支平衡决定, 而不再需要为每个植物功能型单 独设定最大叶面积指数。能量平衡过程模拟的最大 改善在于零平面位移、表面粗糙度等对陆气能量和 水分交换有着重要影响的参数都动态依赖于冠层结 构, 使得模型能够精确刻画冠层变化导致的气候反 馈效应(Ryder et al., 2016)。

(4)森林管理

模型对森林管理过程的模拟建立在解析森林密 度、径级分布和冠层结构的基础上。目前 ORCHIDEE-CAN可以模拟自疏、间伐、皆伐、实生 林和短期萌生林等管理模式。对于非人为管理的森 林(天然林), 自疏主导森林死亡率, 当密度超过根 据Reineke法则计算获得的最大密度时, 直径较小 的树木优先死亡以降低森林密度。模拟实生林时, 先根据相对密度指数进行规律间隔的间伐, 当森林
密度降至某一阈值以下时, 进行皆伐。间伐或皆伐 收获的木材进入木产品碳库进行周转, 枝、叶、树 根等进入凋落物库进行分解。萌生林的采伐取决于 树木直径大小, 采伐时根系生物量保持完好, 模拟 萌生过程。

\subsection{2 草地管理模块}

全球37\% (30\%-47\%)的陆地被草地覆盖, 其中 很大一部分被用于进行畜牧业生产。ORCHIDEEGM模型版本(Chang et al., 2013)融入了PaSim草地 模型(Riedo et al., 1998; Graux et al., 2011)的管理模 块, 用以模拟草料收割、放牧、施肥等过程, 并考虑 这些过程对光合速率(通过施肥), 叶面积指数, 叶 龄, 生物量(通过吃草、踩踏等), 生物量储存、分配 及再生长等的影响。改进后的模型可以用于模拟气 候和草地管理强度变化下区域及全球草地碳平衡。

\subsection{3 农田管理模块}

农田覆盖了超过 $11 \%$ 的陆地表面。农田作为保 障全球粮食安全的重要基础, 受到气候变化和土地 利用变化的强烈影响。同时, 农田开发和利用也对 气候系统产生了重要影响。农田 $\mathrm{N}_{2} \mathrm{O}$ 和甲烷排放以 及泥炭地开发造成的 $\mathrm{CO}_{2}$ 排放已占人为温室气体排 放的 4.5\% (Carlson et al., 2017)。另外, 农业活动灌 溉消耗了全球近 $70 \%$ 的总用水量, 极大程度地改变 了水循环格局。ORCHIDEE-CROP模型版本(Wu et al., 2016)融入了农作物模型STICS (Brisson et al., 1998)的作物生长及管理模块, 考虑小麦、玉米、大 豆、水稻等主要作物的发育阶段和物候等生理过程, 以及播种及收获日期、施肥、灌溉、收获指数等管 理模式, 从而模拟不同管理模式下作物产量对气候 变化的响应以及对农田碳水循环的影响。

另外, ORCHIDEE模型针对冻土(Guimberteau et al., 2018), 泥炭地(Qiu et al., 2018), 有机和无机 碳通过河流的横向输送等过程(Bowring et al., 2019) 发展了不同的版本, 具体过程可以参考相应文章。 不同陆地生物圈模型在所涵盖的过程和模拟机制上 有所不同, 具体的模型过程可以参考附录 I当前主 要陆地生物圈模型的文献列表和Huntzinger等(2013) 正文及其附录表1-4总结的模型过程具体算法。

\section{3 陆地生物圈模型的应用}

随着全球变化生态学的发展, 陆地生物圈模型 在大尺度碳循环、氮循环、水资源、陆气相互作用 
等多个领域有广泛的应用。限于篇幅, 本节主要从 全球变化与碳循环、水循环以及人类活动对生态系 统的影响三方面研究介绍陆地生物圈模型的应用和 进展。这些应用和进展大都基于国际多模型比较计 划的多模型模拟结果, 多模型比较计划的应用和进 展可参考各计划的网站, 例如, M s T M I P (nacp.ornl.gov/MsTMIP.shtml), TRENDY (www. globalcarbonproject.org/)和C4MIP (c4mip.net)。

\section{1 陆地生态系统碳循环}

近 40年陆地碳循环受到科学界和各国政府的广 泛关注, 陆地碳源汇功能对全球变化如何响应是国 际科学界面临的挑战之一。大尺度陆地生产力和碳 源汇功能的评估及其机制分析直接驱动了陆地生物 圈模型中与碳循环有关的过程的发展, 同时陆地生 物圈模型也被直接用于陆地生态系统碳循环的估 算、归因和预测研究。全球碳计划自2006年以来, 每 年发布了基于多个陆地生物圈模拟估算的全球陆地 碳源汇和土地利用碳排放结果(Le Quéré et al., 2018)。基于国际多模型比较计划MsTMIP中多个陆 地生物圈模型模拟结果, Huntzinger等(2017)报道了 大气 $\mathrm{CO}_{2}$ 浓度上升是过去50年陆地碳汇的主要原因, 气候变化和土地利用削弱了陆地碳汇, 氮沉降贡献 了(18 \pm 12)\%的陆地碳汇。基于TRENDY中多个陆 地生物圈模型模拟结果, Zhu等(2016)对近30年遥感 观测到地球变绿进行归因, 发现大气 $\mathrm{CO}_{2}$ 浓度上升 是地球变绿的主要原因。陆地生物圈模型也被用于 研究未来气候变化情境下冻土碳循环的变化 (McGuire et al., 2018)与陆地生产力和碳源汇的变 化(IPCC, 2013)。虽然陆地生物圈模型近年来发展迅 速, 但是陆地生物圈模型对碳循环的模拟仍有很大 的不确定性, 特别是在氮磷与碳的交互作用、生态 系统对极端气候事件的响应、 $\mathrm{CH}_{4}$ 和 $\mathrm{N}_{2} \mathrm{O}$ 等温室气体 排放与吸收、冻土、微生物和植物对气候变化适应 性等方面陆地生物圈模型研究还很薄弱。准确预测 未来陆地碳循环仍需要加强陆地生物圈模型对关键 过程的准确刻画(Prentice \& Cowling, 2013)。

\section{2 陆地生态系统水循环}

陆地生物圈模型对水文过程的模拟可以用来识 别历史径流变化的影响因素(气候、人类活动、植被 变化等), 预估水文过程对未来气候变化和人类活 动的响应, 探讨合理的水资源管理策略等。Piao等 (2007)利用ORCHIDEE模型研究了气候、土地利用
变化和 $\mathrm{CO}_{2}$ 浓度升高对 20 世纪全球陆地径流量变化 的影响, 发现 $\mathrm{CO}_{2}$ 浓度增加导致的叶面积指数 $(L A I)$ 增加降低了径流, 而土地利用变化和气候的影响超 过了 $\mathrm{CO}_{2}$ 的作用, 增加了径流。Yang等(2015)将7个 陆地生物圈模型模拟的地表径流与同一个流量演算 模型耦合起来, 对全球16个大型流域的模拟进行了 评估, 发现模型在低纬度和中纬度地区对河流流量 季节变化的模拟较好, 而在高纬度地区低估了由于 冰雪融化导致的洪峰流量。模拟低估了近30年来流 量的变化趋势, 但多数模型能够解释河流流量年际 波动的 $50 \%$ 以上。土地利用变化对流量的季节变化 和均值影响较弱, 但对长期趋势具有显著影响。

热带地区降水量大, 准确模拟地表水文过程对 于预测未来气候变化非常重要。Santini和Caporaso (2018)评估了 20 个CMIP5地球系统模式中的陆地生 物圈模型对刚果河流量的模拟情况。他们发现模型 可以较好地模拟月和年尺度上河流流量对于平均状 态的偏离, 但是普遍高估了季节变化, 特别是低估 了枯水期的水量。Guimberteau等(2017)发现在未来 全球变暖和 $\mathrm{CO}_{2}$ 浓度升高情形下, 亚马孙流域的降 水量、蒸散和地表径流都呈增加趋势, 而三者在旱 季呈降低趋势; 未来土地利用变化将进一步强化旱 季蒸散减少的趋势, 使得径流增加。此外, 干扰通过 影响植被状态, 也可以影响陆地水文过程。 $\mathrm{Li}$ 和 Lawrence (2017)利用陆地生物圈CLM模型和包含 其在内的CESM地球系统模式研究了野火干扰对陆 地水循环的影响, 发现野火干扰降低了地表蒸散, 增加了地表径流, 但是对降水几乎没有影响。

\section{3 人类活动对陆地生态系统影响的评估}

土地利用和土地管理强度的变化影响生物地球 化学循环并通过改变地表状态产生生物物理反馈, 历史时期土地利用变化贡献了约 $25 \%$ 的人为碳排放 (Le Quéré et al., 2018), 但对区域气候的影响仍有待 进一步评估(Pitman et al., 2009)。未来随着人口增加, 土地资源将变得更加稀缺, 如何在不同利用类型间 分配土地资源以及能否通过土地管理减缓气候变化, 这些都是应对气候变化需要回答的科学问题。国际 上在CMIP6框架下发起了土地利用模型比较计划 (Land Use Model Intercomparison Project, LUMIP), 以推动不同陆地生物圈模型使用相同的驱动数据和 模拟方法, 回答气候政策中与土地利用和管理密切 相关的科学问题(Lawrence et al., 2016)。 
多个融合了农作物模块的陆地生物圈模型 (CLM-crop, LPJ-GUESS, LPJmL, ORCHIDEE-crop) 深入参与了全球格点作物模型比较计划(The Global Gridded Crop Model Inter-comparison (GGCMI); Elliott et al., 2015)。该计划旨在评估现有作物模型 所模拟的作物产量、环境影响等对气候变化、大气 $\mathrm{CO}_{2}$ 含量升高、灌溉和施肥的响应, 分析不确定性来 源, 并探讨模型发展方向(Muller et al., 2017), 为准 确评估未来气候变化和人为管理下作物产量、减产 风险和相关粮食安全等问题提供科学参考。

融入了草地管理模块的陆地生物圈模型(DLEM, LPJ-GUESS, LPJmL, ORCHIDEE) 已被用于定量评 估全球及区域牧草生产潜力对气候变化、大气 $\mathrm{CO}_{2}$ 含量升高、草地利用强度等因子的响应(Chang et al., 2015; Dangal et al., 2016; Rolinski et al., 2018), 模拟 区域草地碳动态及温室气体排放(Chang et al., 2015, 2017; Blanke et al., 2018), 以及对草地管理模式和 利用强度进行优化(Chang et al., 2015; Rolinski et al., 2018)和重建(Chang et al., 2016)。ORCHIDEE-GM模 型还被用于探讨末次冰期猛犸草原大型食草动物生 物量动态及其影响下的草地碳平衡 (Zhu et al., 2018)。

\section{4 展望}

近20年, 陆地生物圈模型成为研究生态系统能 量流动和元素循环的重要方法, 也是地球系统模式 的重要部分。然而, 限于对复杂的陆地生态系统的 有限认知和陆地生物圈模型对陆地生态系统结构和 过程的简单抽象, 如何提高陆地生物圈模型对大尺 度陆地生物圈的模拟精度, 降低模型不确定性是当 前的研究重点和前沿。以下列举了当前陆地生物圈 模型的几个重点发展方向。

\section{1 碳氮磷交互作用}

研究发现大气 $\mathrm{CO}_{2}$ 浓度上升对植被生长的施肥 效应会受到氮磷营养元素的限制(Norby et al., 2010; Vitousek et al., 2010; Terrer et al., 2018), 从而可能 限制未来陆地碳汇强度, 同时增加模型对未来陆地 生态系统碳平衡评估的不确定性(Goll et al., 2012; Zaehle et al., 2015)。多个陆地生物圈模型在已有碳 循环的基础上耦合了氮循环过程, 但已有的模型对 碳氮耦合模拟的不确定性仍然很大, 改进模型碳氮 耦合是模型研究的挑战(Zaehle et al., 2014)。磷循环
也会影响植被生长等碳循环过程, 在陆地生物圈模 型中耦合磷循环也是目前模型的研究热点(Goll et al., 2017; Fleischer et al., 2019)。

\section{2 土壤物理和微生物过程模拟}

土壤团聚体等物理过程对土壤物理和碳氮磷循 环过程有着重要影响(Lehmann \& Kleber, 2015; Sokol et al., 2019), 但目前模型仍缺乏对土壤团聚 体形成等物理过程的模拟(Bradford et al., 2016)。土 壤微生物对土壤有机碳的分解和形成至关重要。研 究发现在陆地生物圈模型中耦合土壤微生物过程可 能会降低陆地对气候变化的正反馈强度(Wieder et al., 2013), 但这一研究结果不确定性很大, 需要进 一步研究。目前如何在陆地生物圈模型中模拟土壤 物理过程和微生物过程及其对生态系统碳氮磷循环 的影响是当前的研究热点和难点 (Tang \& Riley, 2017; Wieder et al., 2018)。

\section{3 人类活动的模拟}

地球陆地表层 $42 \%-68 \%$ 的面积受人类活动影 响, 人类对森林、草地和农田的经营管理, 如人工林 管理、木材采集、放牧、割草、施肥、灌溉等措施 直接影响了生态系统结构和功能。另外, 迁徙耕作、 耕地过度开唇、过度放牧、泥炭地开发(包括火干扰) 以及气候变化(全球变暖、降水强度变化、干旱等) 可能造成土地退化(水蚀、风蚀、营养流失、质地变 化)并对生态系统碳循环产生显著影响。随着人类对 生态系统的影响日益加剧, 如何在陆地生物圈模型 中模拟人类活动也是当前的研究热点和难点(Fisher et al., 2014; Naudts et al., 2016)。

\section{4 森林干扰模拟}

干扰(火灾、风灾、虫灾、雪灾、干旱、病原性 灾害等)是森林生态系统的固有组成部分, 对森林 生态系统造成破坏性影响, 可以改变森林结构、物 种组成并影响其生态系统服务功能。全球变化既可 以通过气候变化影响干扰发生的频率、范围和强度, 又可以通过影响植被组成间接影响干扰的发生 (Seidl et al., 2017)。然而, 干扰在陆地生物圈模型中 的表征仍不完善。目前多数模型包括了基于机制的 火灾模块(Rabin et al., 2017), 然而对于其他形式干 扰的模拟仍普遍欠缺。模拟某些自然灾害(如风灾)、 以及模拟多重干扰的相互作用需要解析森林冠层结 构, 以表征灾害过后冠层结构变化对次生灾害发生 的影响。可以预见，不断完善对森林干扰的模拟将 
是下一阶段模型发展的一个重点方向。

\section{5 模型-数据融合}

随着观测数据的积累和大数据时代的来临, 如 何利用不同时空尺度的观测数据提高模型的模拟精 度也是当前的研究热点和难点(Prentice \& Cowling, 2013; Medlyn et al., 2015)。如何降低陆地生物圈模 型的不确定性是科学界面临的巨大挑战。陆地生物 圈模型的不确定性主要来源于模型结构、过程、参 数、气候和土壤驱动数据。基于观测数据和数据模 型融化算法优化模型参数(Kuppel et al., 2014; Bastrikov et al., 2018)可以显著改进陆地生物圈模型的 模拟精度。全球越来越多从叶片到生态系统尺度的 观测数据和全球变化控制实验等数据为模型参数优 化和模型数据融合提供了基础(Prentice \& Cowling, 2013)。但目前观测数据和全球变化控制实验主要集 中在温带和寒带, 降低陆地生物圈模型在热带模拟 的不确定性需要加强热带的观测和模型数据融合研 究(Fleischer et al., 2019)。

\section{参考文献}

Bastrikov V, MacBean N, Bacour C, Santaren D, Kuppel S, Peylin P (2018). Land surface model parameter optimisation using in situ flux data: comparison of gradient-based versus random search algorithms (a case study using ORCHIDEE v1.9.5.2). Geoscientific Model Development, 11, 4739-4754.

Blanke J, Boke-Olén N, Olin S, Chang JF, Sahlin U, Lindeskog M, Lehsten V (2018). Implications of accounting for management intensity on carbon and nitrogen balances of European grasslands. PLOS ONE, 13, e0201058. DOI: 10.1371/journal.pone.0201058.

Bodman RW, Rayner PJ, Karoly DJ (2013). Uncertainty in temperature projections reduced using carbon cycle and climate observations. Nature Climate Change, 3, 725-729.

Bowring SPK, Lauerwald R, Guenet B, Zhu D, Guimberteau M, Tootchi A, Ducharne A, Ciais P (2019). ORCHIDEE MICT-LEAK (r5459), a global model for the production, transport, and transformation of Dissolved organic carbon from Arctic permafrost regions-Part 1: Rationale, model description, and simulation protocol. Geoscientific Model Development, 12, 3503-3521.

Bradford MA, Wieder WR, Bonan GB, Fierer N, Raymond PA, Crowther TW (2016). Managing uncertainty in soil carbon feedbacks to climate change. Nature Climate Change, 6, 751-758.

Brisson N, Mary B, Ripoche D, Jeuffroy MH, Ruget F, Nicoullaud B, Gate P, Devienne-Barret F, Antonioletti R, Durr C,
Richard G, Beaudoin N, Recous S, Tayot X, Plenet D, Cellier P, MacHet JM, Meynard JM, Delécolle R (1998). STICS: a generic model for the simulation of crops and their water and nitrogen balances. I. Theory and parameterization applied to wheat and corn. Agronomie, 18, 311-346.

Carlson KM, Gerber JS, Mueller ND, Herrero M, MacDonald GK, Brauman KA, Havlik P, O’Connell CS, Johnson JA, Saatchi S, West PC (2017). Greenhouse gas emissions intensity of global croplands. Nature Climate Change, 7, 63-68.

Chang JF, Ciais P, Herrero M, Havlik P, Campioli M, Zhang XZ, Bai YF, Viovy N, Joiner J, Wang XH, Peng SS, Yue C, Piao SL, Wang T, Hauglustaine DA, Soussana JF, Peregon A, Kosykh N, Mironycheva-Tokareva N (2016). Combining livestock production information in a processbased vegetation model to reconstruct the history of grassland management. Biogeosciences, 13, 3757-3776.

Chang JF, Ciais P, Viovy N, Soussana JF, Klumpp K, Sultan B (2017). Future productivity and phenology changes in European grasslands for different warming levels: implications for grassland management and carbon balance. Carbon Balance and Management, 12. DOI: 10.1186/ s13021-017-0079-8.

Chang JF, Ciais P, Viovy N, Vuichard N, Sultan B, Soussana JF (2015). The greenhouse gas balance of European grasslands. Global Change Biology, 21, 3748-3761.

Chang JF, Viovy N, Vuichard N, Ciais P, Wang T, Cozic A, Lardy R, Graux AI, Klumpp K, Martin R, Soussana JF (2013). Incorporating grassland management in ORCHIDEE: model description and evaluation at 11 eddy-covariance sites in Europe. Geoscientific Model Development, 6, 2165-2181.

Dangal SRS, Tian HQ, Lu CQ, Pan SF, Pederson N, Hessl A (2016). Synergistic effects of climate change and grazing on net primary production of Mongolian grasslands. Ecosphere, 7, e01274. DOI: 10.1002/ecs2.1274.

de Rosnay P, Polcher J (1998). Modelling root water uptake in a complex land surface scheme coupled to a GCM. Hydrology and Earth System Sciences, 2, 239-255.

de Rosnay P, Polcher J, Bruen M, Laval K (2002). Impact of a physically based soil water flow and soil-plant interaction representation for modeling large-scale land surface processes. Journal of Geophysical Research, 107, 4118. DOI: 10.1029/2001JD000634.

Ducoudré NI, Laval K, Perrier A (1993). SECHIBA, a new set of parameterizations of the hydrologic exchanges at the land-atmosphere interface within the LMD atmospheric general circulation model. Journal of Climate, 6, 248-273.

Elliott J, Müller C, Deryng D, Chryssanthacopoulos J, Boote KJ, Büchner M, Foster I, Glotter M, Heinke J, Iizumi T, Izaurralde RC, Mueller ND, Ray DK, Rosenzweig C,

www.plant-ecology.com 
Ruane AC, Sheffield J (2015). The Global Gridded Crop Model Intercomparison: data and modeling protocols for Phase 1 (v1.0). Geoscientific Model Development, 8, 261-277.

Fisher JB, Huntzinger DN, Schwalm CR, Sitch S (2014). Modeling the terrestrial biosphere. Annual Review of Environment and Resources, 39, 91-123.

Fleischer K, Rammig A, de Kauwe MG, Walker AP, Domingues TF, Fuchslueger L, Garcia S, Goll DS, Grandis A, Jiang MK, Haverd V, Hofhansl F, Holm JA, Kruijt B, Leung F, Medlyn BE, Mercado LM, Norby RJ, Pak B, von Randow C, Quesada CA, Schaap KJ, Valverde-Barrantes OJ, Wang YP, Yang XJ, Zaehle S, Zhu Q, Lapola DM (2019). Amazon forest response to $\mathrm{CO}_{2}$ fertilization dependent on plant phosphorus acquisition. Nature Geoscience, 12, 736-741.

Friedlingstein P, Cox P, Betts R, Bopp L, von Bloh W, Brovkin V, Cadule P, Doney S, Eby M, Fung I, Bala G, John J, Jones C, Joos F, Kato T, Kawamiya M, Knorr W, Lindsay K, Matthews HD, Raddatz T, Rayner P, Reick C, Roeckner E, Schnitzler KG, Schnur R, Strassmann K, Weaver AJ, Yoshikawa C, Zeng N (2006). Climate-carbon cycle feedback analysis: results from the C4MIP model intercomparison. Journal of Climate, 19, 3337-3353.

Friedlingstein P, Meinshausen M, Arora VK, Jones CD, Anav A, Liddicoat SK, Knutti R (2014). Uncertainties in CMIP5 climate projections due to carbon cycle feedbacks. Journal of Climate, 27, 511-526.

Friend AD, Stevens AK, Knox RG, Cannell MGR (1997). A process-based, terrestrial biosphere model of ecosystem dynamics (Hybrid v3.0). Ecological Modelling, 95, 249-287.

Goll DS, Brovkin V, Parida BR, Reick CH, Kattge J, Reich PB, van Bodegom PM, Niinemets Ü (2012). Nutrient limitation reduces land carbon uptake in simulations with a model of combined carbon, nitrogen and phosphorus cycling. Biogeosciences, 9, 3547-3569.

Goll DS, Vuichard N, Maignan F, Jornet-Puig A, Sardans J, Violette A, Peng SS, Sun Y, Kvakic M, Guimberteau M, Guenet B, Zaehle S, Penuelas J, Janssens I, Ciais P (2017). A representation of the phosphorus cycle for ORCHIDEE (revision 4520). Geoscientific Model Development, 10, 3745-3770.

Graux AI, Gaurut M, Agabriel J, Baumont R, Delagarde R, Delaby L, Soussana JF (2011). Development of the Pasture Simulation Model for assessing livestock production under climate change. Agriculture, Ecosystems \& Environment, 144, 69-91.

Guimberteau M, Ciais P, Ducharne A, Boisier JP, Dutra Aguiar AP, Biemans H, de Deurwaerder H, Galbraith D, Kruijt B, Langerwisch F, Poveda G, Rammig A, Rodriguez DA, Tejada G, Thonicke K, von Randow C, von Randow RCS,
Zhang K, Verbeeck H (2017). Impacts of future deforestation and climate change on the hydrology of the Amazon Basin: a multi-model analysis with a new set of land-cover change scenarios. Hydrology and Earth System Sciences, 21, 1455-1475.

Guimberteau M, Zhu D, Maignan F, Huang Y, Yue C, DantecNédélec $\mathrm{S}$, Ottlé $\mathrm{C}$, Jornet-Puig $\mathrm{A}$, Bastos $\mathrm{A}$, Laurent $\mathrm{P}$, Goll D, Bowring S, Chang J, Guenet B, Tifafi M, Peng S, Krinner G, Ducharne A, Wang F, Wang T, Wang X, Wang Y, Yin Z, Lauerwald R, Joetzjer E, Qiu C, Kim H, Ciais P (2018). ORCHIDEE-MICT (v8.4.1), a land surface model for the high latitudes: model description and validation. Geoscientific Model Development, 11, 121-163.

Huntzinger DN, Michalak AM, Schwalm C, Ciais P, King AW, Fang Y, Schaefer K, Wei Y, Cook RB, Fisher JB, Hayes D, Huang M, Ito A, Jain AK, Lei H, Lu C, Maignan F, Mao J, Parazoo N, Peng S, Poulter B, Ricciuto D, Shi X, Tian H, Wang W, Zeng N, Zhao F (2017). Uncertainty in the response of terrestrial carbon sink to environmental drivers undermines carbon-climate feedback predictions. Scientific Reports, 7, 4765. DOI: 10.1038/s41598-01703818-2.

Huntzinger DN, Schwalm C, Michalak AM, Schaefer K, King AW, Wei Y, Jacobson A, Liu S, Cook RB, Post WM, Berthier G, Hayes D, Huang M, Ito A, Lei H, Lu C, Mao J, Peng CH, Peng S, Poulter B, Riccuito D, Shi X, Tian H, Wang W, Zeng N, Zhao F, Zhu Q (2013). The north American carbon program multi-scale synthesis and terrestrial model intercomparison project-Part 1: overview and experimental design. Geoscientific Model Development, 6, 2121-2133.

IPCC (2013). Climate Change 2013: the Physical Science Basis. Cambridge University Press, Cambridge, UK.

Krinner G, Viovy N, de Noblet-Ducoudré N, Ogée J, Polcher J, Friedlingstein P, Ciais P, Sitch S, Prentice IC (2005). A dynamic global vegetation model for studies of the coupled atmosphere-biosphere system. Global Biogeochemical Cycles, 19, GB1015. DOI: 10.1029/2003gb002199.

Kuppel S, Peylin P, Maignan F, Chevallier F, Kiely G, Montagnani L, Cescatti A (2014). Model-data fusion across ecosystems: from multisite optimizations to global simulations. Geoscientific Model Development, 7, 2581-2597.

Lawrence DM, Hurtt GC, Arneth A, Brovkin V, Calvin KV, Jones AD, Jones CD, Lawrence PJ, de Noblet-Ducoudré N, Pongratz J, Seneviratne SI, Shevliakova E (2016). The Land Use Model Intercomparison Project (LUMIP) contribution to CMIP6: rationale and experimental design. Geoscientific Model Development, 9, 2973-2998.

Le Quéré C, Andrew RM, Friedlingstein P, Sitch S, Hauck J, Pongratz J, Pickers PA, Korsbakken JI, Peters GP, Canadell JG, Arneth A, Arora VK, Barbero L, Bastos A, Bopp L, Chevallier F, Chini LP, Ciais P, Doney SC, 
Gkritzalis T, Goll DS, Harris I, Haverd V, Hoffman FM, Hoppema M, Houghton RA, Hurtt G, Ilyina T, Jain AK, Johannessen T, Jones CD, Kato E, Keeling RF, Goldewijk KK, Landschützer $\mathrm{P}$, Lefèvre $\mathrm{N}$, Lienert $\mathrm{S}$, Liu $\mathrm{Z}$, Lombardozzi D, Metzl N, Munro DR, Nabel JEMS, Nakaoka SI, Neill C, Olsen A, Ono T, Patra P, Peregon A, Peters W, Peylin P, Pfeil B, Pierrot D, Poulter B, Rehder G, Resplandy L, Robertson E, Rocher M, Rödenbeck C, Schuster U, Schwinger J, Séférian R, Skjelvan I, Steinhoff T, Sutton A, Tans PP, Tian HQ, Tilbrook B, Tubiello FN, van der Laan-Luijkx IT, van der Werf GR, Viovy N, Walker AP, Wiltshire AJ, Wright R, Zaehle S, Zheng B (2018). Global carbon budget 2018. Earth System Science Data, 10, 2141-2194.

Lehmann J, Kleber M (2015). The contentious nature of soil organic matter. Nature, 528, 60-68.

Li F, Lawrence DM (2017). Role of fire in the global land water budget during the twentieth century due to changing ecosystems. Journal of Climate, 30, 1893-1908.

McGuire AD, Lawrence DM, Koven C, Clein JS, Burke E, Chen GS, Jafarov E, MacDougall AH, Marchenko S, Nicolsky D, Peng SS, Rinke A, Ciais P, Gouttevin I, Hayes DJ, Ji DY, Krinner G, Moore JC, Romanovsky V, Schädel C, Schaefer K, Schuur EAG, Zhuang QL (2018). Dependence of the evolution of carbon dynamics in the northern permafrost region on the trajectory of climate change. Proceedings of the National Academy of Sciences of the United States of America, 115, 3882-3887.

Medlyn BE, Zaehle S, de Kauwe MG, Walker AP, Dietze MC, Hanson PJ, Hickler T, Jain AK, Luo YQ, Parton W, Prentice IC, Thornton PE, Wang SS, Wang YP, Weng ES, Iversen CM, McCarthy HR, Warren JM, Oren R, Norby RJ (2015). Using ecosystem experiments to improve vegetation models. Nature Climate Change, 5, 528-534.

Moorcroft PR, Hurtt GC, Pacala SW (2001). A method for scaling vegetation dynamics: the ecosystem demography model (ED). Ecological Monographs, 71, 557-586.

Muller A, Schader C, El-Hage Scialabba N, Brüggemann J, Isensee A, Erb KH, Smith P, Klocke P, Leiber F, Stolze M, Niggli U (2017). Strategies for feeding the world more sustainably with organic agriculture. Nature Communications, 8, 1290. DOI: 10.1038/s41467-017-01410-w.

Naudts K, Chen Y, McGrath MJ, Ryder J, Valade A, Otto J, Luyssaert S (2016). Europes forest management did not mitigate climate warming. Science, 351, 597-600.

Norby RJ, Warren JM, Iversen CM, Medlyn BE, McMurtrie $\mathrm{RE}$ (2010). $\mathrm{CO}_{2}$ enhancement of forest productivity constrained by limited nitrogen availability. Proceedings of the National Academy of Sciences of the United States of America, 107, 19368-19373.

Parton WJ, Ojima DS, Cole CV, Schimel DS (1994). A General Model for Soil Organic Matter Dynamics: Sensitivity to
Litter Chemistry, Texture and Management. Quantitative Modeling of Soil Forming Processes. Soil Science Society of America, Madison, Wisconsin, USA.

Piao S, Friedlingstein P, Ciais P, de Noblet-Ducoudré N, Labat $\mathrm{D}$, Zaehle S (2007). Changes in climate and land use have a larger direct impact than rising $\mathrm{CO}_{2}$ on global river runoff trends. Proceedings of the National Academy of Sciences of the United States of America, 104, 15242-15247.

Pitman AJ, de Noblet-Ducoudré N, Cruz FT, Davin EL, Bonan GB, Brovkin V, Claussen M, Delire C, Ganzeveld L, Gayler V, van den Hurk BJJM, Lawrence PJ, van der Molen MK, Müller C, Reick CH, Seneviratne SI, Strengers BJ, Voldoire A (2009). Uncertainties in climate responses to past land cover change: first results from the LUCID intercomparison study. Geophysical Research Letters, 36, L14814. DOI: 10.1029/2009GL039076.

Prentice IC, Bondeau A, Cramer W, Harrison SP, Hickler T, Lucht W, Sitch S, Smith B, Sykes MT (2007). Dynamic Global Vegetation Modeling: Quantifying Terrestrial Ecosystem Responses to Large-scale Environmental Change. Terrestrial Ecosystems in a Changing World. Springer, Berlin.

Prentice IC, Cowling SA (2013). Dynamic global vegetation models//Levin SA. Encyclopedia of Biodiversity. 2nd ed. Elsevier, Amsterdam.

Qiu CJ, Zhu D, Ciais P, Guenet B, Krinner G, Peng SS, Aurela M, Bernhofer C, Brümmer C, Bret-Harte S, Chu HS, Chen JQ, Desai AR, Dušek J, Euskirchen ES, Fortuniak K, Flanagan LB, Friborg T, Grygoruk M, Gogo S, Grünwald T, Hansen BU, Holl D, Humphreys E, Hurkuck M, Kiely G, Klatt J, Kutzbach L, Largeron C, Laggoun-Défarge F, Lund M, Lafleur PM, Li XF, Mammarella I, Merbold L, Nilsson MB, Olejnik J, Ottosson-Löfvenius M, Oechel W, Parmentier FJW, Peichl M, Pirk N, Peltola O, Pawlak W, Rasse D, Rinne J, Shaver G, Schmid HP, Sottocornola M, Steinbrecher R, Sachs T, Urbaniak M, Zona D, Ziemblinska K (2018). ORCHIDEE-PEAT (revision 4596), a model for northern peatland $\mathrm{CO}_{2}$, water, and energy fluxes on daily to annual scales. Geoscientific Model Development, 11, 497-519.

Rabin SS, Melton JR, Lasslop G, Bachelet D, Forrest M, Hantson S, Kaplan JO, Li F, Mangeon S, Ward DS, Yue C, Arora VK, Hickler T, Kloster S, Knorr W, Nieradzik L, Spessa A, Folberth GA, Sheehan T, Voulgarakis A, Kelley DI, Prentice IC, Sitch S, Harrison S, Arneth A (2017). The Fire Modeling Intercomparison Project (FireMIP), phase 1: experimental and analytical protocols with detailed model descriptions. Geoscientific Model Development, 10, 1175-1197.

Riedo M, Grub A, Rosset M, Fuhrer J (1998). A pasture simulation model for dry matter production, and fluxes of carbon, nitrogen, water and energy. Ecological Modelling,

www.plant-ecology.com 
105, 141-183.

Rolinski S, Müller C, Heinke J, Weindl I, Biewald A, Bodirsky BL, Bondeau A, Boons-Prins ER, Bouwman AF, Leffelaar PA, te Roller JA, Schaphoff S, Thonicke K (2018). Modeling vegetation and carbon dynamics of managed grasslands at the global scale with LPJmL 3.6. Geoscientific Model Development, 11, 429-451.

Rothermel RC (1972). A Mathematical Model for Predicting Fire Spread in Wildland Fuels. USDA Forest Service General Technical Report INT-115. Intermountain Forest and Range Experiment Station, Forest Service, US Dept. of Agriculture, Ogden, Utah.

Ryder J, Polcher J, Peylin P, Ottlé C, Chen Y, van Gorsel E, Haverd V, McGrath MJ, Naudts K, Otto J, Valade A, Luyssaert S (2016). A multi-layer land surface energy budget model for implicit coupling with global atmospheric simulations. Geoscientific Model Development, 9, 223-245.

Santini M, Caporaso L (2018). Evaluation of freshwater flow from rivers to the sea in CMIP5 simulations: insights from the Congo river basin. Journal of Geophysical Research, 123, 10278-10300.

Seidl R, Thom D, Kautz M, Martin-Benito D, Peltoniemi M, Vacchiano G, Wild J, Ascoli D, Petr M, Honkaniemi J, Lexer MJ, Trotsiuk V, Mairota P, Svoboda M, Fabrika M, Nagel TA, Reyer CPO (2017). Forest disturbances under climate change. Nature Climate Change, 7, 395-402.

Sellers PJ, Dickinson RE, Randall DA, Betts AK, Hall FG, Berry JA, Collatz GJ, Denning AS, Mooney HA, Nobre CA, Sato N, Field CB, Henderson-Sellers A (1997). Modeling the exchanges of energy, water, and carbon between continents and the atmosphere. Science, 275, 502-509.

Sitch S, Friedlingstein P, Gruber N, Jones SD, MurrayTortarolo G, Ahlström A, Doney SC, Graven H, Heinze C, Huntingford C, Levis S, Levy PE, Lomas M, Poulter B, Viovy N, Zaehle S, Zeng N, Arneth A, Bonan G, Bopp L, Canadell JG, Chevallier F, Ciais P, Ellis R, Gloor M, Peylin P, Piao SL, Le Quéré C, Smith B, Zhu Z, Myneni R (2015). Recent trends and drivers of regional sources and sinks of carbon dioxide. Biogeosciences, 12, 653-679.

Sitch S, Smith B, Prentice IC, Arneth A, Bondeau A, Cramer W, Kaplan JO, Levis S, Lucht W, Sykes MT, Thonicke K, Venevsky S (2003). Evaluation of ecosystem dynamics, plant geography and terrestrial carbon cycling in the LPJ dynamic global vegetation model. Global Change Biology, 9, 161-185.

Sokol NW, Sanderman J, Bradford MA (2019). Pathways of mineral-associated soil organic matter formation: integrating the role of plant carbon source, chemistry, and point of entry. Global Change Biology, 25, 12-24.

Tang JY, Riley WJ (2017). SUPECA kinetics for scaling redox reactions in networks of mixed substrates and consumers and an example application to aerobic soil respiration. Geoscientific Model Development, 10, 3277-3295.

Terrer C, Vicca S, Stocker BD, Hungate BA, Phillips RP, Reich PB, Finzi AC, Prentice IC (2018). Ecosystem responses to elevated $\mathrm{CO}_{2}$ governed by plant-soil interactions and the cost of nitrogen acquisition. New Phytologist, 217, 507-522.

Vitousek PM, Porder S, Houlton BZ, Chadwick OA (2010). Terrestrial phosphorus limitation: mechanisms, implications, and nitrogen-phosphorus interactions. Ecological Applications, 20, 5-15.

Watt AS (1947). Pattern and process in the plant community. Journal of Ecology, 35, 1-22.

Wieder WR, Bonan GB, Allison SD (2013). Global soil carbon projections are improved by modelling microbial processes. Nature Climate Change, 3, 909-912.

Wieder WR, Hartman MD, Sulman BN, Wang YP, Koven CD, Bonan GB (2018). Carbon cycle confidence and uncertainty: exploring variation among soil biogeochemical models. Global Change Biology, 24, 1563-1579.

Wu X, Vuichard N, Ciais P, Viovy N, de Noblet-Ducoudré N, Wang X, Magliulo V, Wattenbach M, Vitale L, di Tommasi P, Moors EJ, Jans W, Elbers J, Ceschia E, Tallec T, Bernhofer C, Grünwald T, Moureaux C, Manise T, Ligne A, Cellier P, Loubet B, Larmanou E, Ripoche D (2016). ORCHIDEE-CROP (v0), a new process-based agro-land surface model: model description and evaluation over Europe. Geoscientific Model Development, 9, 857-873.

Yang H, Piao SL, Zeng ZZ, Ciais P, Yin Y, Friedlingstein P, Sitch S, Ahlström A, Guimberteau M, Huntingford C, Levis S, Levy PE, Huang MT, Li Y, Li XR, Lomas MR, Peylin P, Poulter B, Viovy N, Zaehle S, Zeng N, Zhao F, Wang L (2015). Multicriteria evaluation of discharge simulation in dynamic global vegetation models. Journal of Geophysical Research, 120, 7488-7505.

Yin XY, Struik PC, Romero P, Harbinson J, Evers JB, van der Putten PEL, Vos J (2009). Using combined measurements of gas exchange and chlorophyll fluorescence to estimate parameters of a biochemical $\mathrm{C}_{3}$ photosynthesis model: a critical appraisal and a new integrated approach applied to leaves in a wheat (Triticum aestivum) canopy. Plant, Cell \& Environment, 32, 448-464.

Zaehle S, Jones CD, Houlton B, Lamarque JF, Robertson E (2015). Nitrogen availability reduces CMIP5 projections of twenty-first-century land carbon uptake. Journal of Climate, 28, 2494-2511.

Zaehle S, Medlyn BE, de Kauwe MG, Walker AP, Dietze MC, Hickler T, Luo YQ, Wang YP, El-Masri B, Thornton P, Jain A, Wang SS, Warlind D, Weng ES, Parton W, Iversen CM, Gallet-Budynek A, McCarthy H, Finzi A, Hanson PJ, Prentice IC, Oren R, Norby RJ (2014). Evaluation of 11 terrestrial carbon-nitrogen cycle models against observations from two temperate Free-Air $\mathrm{CO}_{2}$ Enrichment 
studies. New Phytologist, 202, 803-822.

Zhu D, Ciais P, Chang JF, Krinner G, Peng SS, Viovy N, Peñuelas J, Zimov S (2018). The large mean body size of mammalian herbivores explains the productivity paradox during the Last Glacial Maximum. Nature Ecology \& Evolution, 2, 640-649.

Zhu ZC, Piao SL, Myneni RB, Huang MT, Zeng ZZ, Canadell JG, Ciais P, Sitch S, Friedlingstein P, Arneth A, Cao CX,
Cheng L, Kato E, Koven C, Li Y, Lian X, Liu YW, Liu RG, Mao JF, Pan YZ, Peng SS, Peñuelas J, Poulter B, Pugh TAM, Stocker BD, Viovy N, Wang XH, Wang YP, Xiao ZQ, Yang H, Zaehle S, Zeng N (2016). Greening of the earth and its drivers. Nature Climate Change, 6, 791-795.

特邀编委: 温学发 责任编辑: 李 敏

附录I 参与目前国际模型比较计划MsTMIP和TRENDY的陆地生物圈模型及其参考文献

Supplement I Terrestrial biosphere models involved in international model inter-comparison projects (MsTMIP and TRENDY) and their references

http://www.plant-ecology.com/fileup/1005-264X/PDF/cjpe.2019.0315-S1.pdf 